\title{
MEAN-CURVATURE FLOW OF VORONOI DIAGRAMS
}

\author{
MATT ELSEY, DEJAN SLEPČEV
}

\begin{abstract}
We study the evolution of grain boundary networks by the mean-curvature flow under the restriction that the networks are Voronoi diagrams for a set of points. For such evolution we prove a rigorous universal upper bound on the coarsening rate. The rate agrees with the rate predicted for the evolution by mean-curvature flow of the general grain boundary networks, namely that the typical grain area grows linearly in time. We perform numerical simulations which provide evidence that the dynamics achieves the rate of coarsening that agrees with the upper bound in terms of scaling.
\end{abstract}

\section{INTRODUCTION}

The aim of this work is twofold. First, we describe the analogue of mean-curvature flow of a network of curves in the restricted class of networks arising as the Voronoi diagrams (for appropriate set of points). Second, we provide a rigorous, universal, upper bound on the rate of coarsening for the system.

Since the pioneering work of Kohn and Otto [20], rigorous upper bounds on the rate of coarsening have been established in various systems. For example, Kohn and Otto considered the Mullins-Sekerka problem which is the gradient flow of the perimeter in two-phase systems with respect to the $H^{-1}$ norm of the vector field that transports the phases. They show a weak form of the upper bound $\ell \lesssim t^{1 / 3}$ where $\ell$ is the typical length scale of the system.

The evolution of interfaces by mean-curvature flow raises a number of interesting questions. Heuristic arguments based on scaling suggest that if one considers the evolution of labyrinthine patterns under the flow where the normal velocity is equal to the mean curvature then the typical length scale of the system should scale like

$$
\ell \sim t^{1 / 2}
$$

See Glazier [11] for the heuristic argument and a review of early work (prior to Kohn and Otto) attempting to verify this conjecture by numerical, experimental, and mean field approaches. However, if one starts with a family of disjoint balls, the coarsening is over in finite time (independent of the system size), and thus a universal upper bound on the rate cannot hold. Even if one considers the volume-preserving mean-curvature flow (which, like the Mullins-Sekerka evolution, preserves the mass of the phases), the universal upper bound on the coarsening does not hold. On the other hand, there have been partial results on coarsening rates by Dai [7] and Mugnai and Seis [25] which hold provided that the dynamics satisfies some assumptions (which are reasonable if one of the phases is relatively sparse).

A related model of coarsening driven by mean curvature is the evolution of grain boundary networks. Under this model, a system with many phases (instead of two) evolves by mean curvature. This system is particularly relevant to materials science.

Date: August 5, 2013. 
Its investigation started with the works of Beck [4], Herring [14], Mullins [26] and von Neumann [29]. Since then many interesting properties, generalizations and applications have been investigated (see $[3,5,17,18,23]$ and references therein), but fundamental questions such as rigorous results on long-time existence remain open. Extensive numerical simulations $[1,2,8,9,30]$ in $2 \mathrm{D}$ and $3 \mathrm{D}$ show that for generic data the typical area of grains scales linearly with time, but a proof of an upper bound on the rate remains elusive.

Here we study a simplified system where the grain boundary networks are restricted to be Voronoi diagrams for a set of points. We investigate it in two dimensions. We remark that there are no fundamental obstacles to extending our approach to three or more dimensions, only that defining the flow becomes more involved. For the evolution of Voronoi diagrams by mean curvature we prove that a weak form of the bound

$$
\ell \lesssim t^{1 / 2}
$$

holds universally. Our work is inspired by the work of Henseler, Niethammer, and Otto [12], who restricted the configuration space to be the set of planar grain boundary networks with straight edges. They show numerically that such system also has the rate of coarsening $\ell \lesssim t^{1 / 2}$. In the extensive numerical simulations they carried out one can observe that if the grains are initially convex they remain so. When the convexity of grains holds the proof we present for Voronoi diagrams applies and implies an upper bound on the coarsening rate for their model too. However, for special (carefully constructed) configurations the convexity can be violated; and furthermore, there can be interpenetrations of grains. Thus one would need to modify the dynamics a bit to make it well-defined. We do not attempt to do so in this paper. Instead, we further restrict the configuration space to be the set of Voronoi diagrams. This leads to a well-defined system, but also brings a number of challenges.

A Voronoi diagram is typically given by specifying the centers of the cells. However, during the mean-curvature flow of Voronoi diagrams centers of the cells can (and do) collide and remain collided. To allow for this we need to define the configuration space as a manifold with corners and more generally address the issues arising from considering a gradient flow on a manifold with corners. Another issue is that the network can undergo topological changes. In particular, the cell neighborhood structure can change and cells can disappear. Furthermore, the energy of a configuration (total perimeter) is not a smooth function of the positions of the centers of the Voronoi cells when topological changes occur. Finally we needed to modify the Kohn-Otto framework. Namely, we define the length scale $L(t)$, which plays a key role in the framework, not as a function of the configuration at time $t$, but instead depending on the whole solution path up to time $t$. The modification is general and can be applied to other coarsening systems.

Let us remark that there are different simplifications of the evolution of grain boundary networks which have been sucessfully analyzed. Fradkov [10] introduced a meanfield type model for the grain size distribution. Recently, Herrmann, Laurençot, and Niethammer [15] showed that the system admits self-similar solutions.

The paper is laid out as follows: In Section 2, we describe the mean-curvature flow for a general network of curves. The discussion of the restriction of the mean-curvature flow to the subspace of the set of grain boundary networks which are Voronoi diagrams is started in Section 3. In Section 4, we briefly review background on the differential geometry of manifolds with corners, which is needed to describe the restricted configuration space. The description of the gradient flow for Voronoi diagrams is completed 
in Section 5. The universal upper bound on the coarsening rate for this flow is proved in Section 6. Finally, numerical tests suggesting the correctness of our approach and demonstrating agreement with the upper bound on the coarsening rate are presented in Section 7 .

\section{Mean-Curvature Flow of GRAin Boundary NETWORKS}

We begin by defining a grain boundary network and the mean-curvature flow in two dimensions. Let $\mathbb{T}_{2}$ be a flat two-dimensional torus of side length $\Lambda$. A grain boundary network, $\mathcal{G}$, is a finite collection of grains, that is, open connected sets, $G_{i}, i \in I$ with piecewise $C^{1}$ boundaries, that are pairwise disjoint and whose closures fill the whole space:

$$
G_{i} \cap G_{j}=\emptyset \quad \text { if } i \neq j \quad \text { and } \bigcup_{i \in I} \overline{G_{i}}=\mathbb{T}_{2} .
$$

We denote the grain boundaries by $\partial \mathcal{G}=\bigcup_{i \in I} \partial G_{i}$. We define junctions, $\mathcal{J}$, to be the points which belong to boundaries of three or more grains.

We define the energy of a grain boundary network to be the total length of grain boundaries:

$$
\mathcal{E}(\mathcal{G})=\frac{1}{2} \sum_{i \in I} \operatorname{Per}\left(G_{i}\right)
$$

The time evolution of grain boundary networks in which boundaries move continuously and no new grains can be created is described by specifying the normal velocity of the grain boundaries:

$$
\mathcal{V}(\mathcal{G})=\left\{v \in L^{2}\left(\partial \mathcal{G}, \mathbb{R}^{2}\right) \text { : for a.e. } x \in \partial \mathcal{G} \text { if } x \in \partial G_{i} \text { then } v(x) \perp \partial G_{i}\right\} .
$$

On $\mathcal{V}(\mathcal{G})$ we define the inner product $g$ by

$$
g\left(v_{1}, v_{2}\right)=\int_{\partial \mathcal{G}} v_{1} \cdot v_{2} d \mathcal{H}^{1},
$$

where $v_{1} \cdot v_{2}$ indicates the scalar product of vectors $v_{1}$ and $v_{2}$. We say that a family of grain boundary networks $\left\{\mathcal{G}(t): t \in\left[t_{1}, t_{2}\right]\right\}$ evolves by velocity $v: \bigcup_{t \in\left[t_{1}, t_{2}\right]}\{t\} \times \partial \mathcal{G}(t) \rightarrow$ $\mathbb{R}^{2}$ if there exists a continuous family of homeomorphisms $\Phi(t): \partial \mathcal{G}\left(t_{1}\right) \rightarrow \partial \mathcal{G}(t)$ such that $\frac{d \Phi(t)}{d t}(x)=v(x, t)$ for all $t$ and a.e. $x$.

Given a junction $x \in \mathcal{J}$ which is an intersection of $m$ (closures of) grains, there exist $\varepsilon>0$ and $m$ arc-length parameterized curves $\gamma_{i}:[0, \varepsilon) \rightarrow \mathbb{T}_{2}$ such that $\gamma_{i}(0)=x$ and that $\partial \mathcal{G} \cap B(x, r)=\bigcup_{i=1}^{m} \gamma_{i}(([0, \varepsilon)) \cap B(x, r)$, for $r>0$ small enough. We define $b(x)=\sum_{i=1}^{m} \gamma_{i}^{\prime}(0)$.

Given a family of grain boundary networks $\left\{\mathcal{G}(t): t \in\left[t_{1}, t_{2}\right]\right\}$ evolving by a continuous velocity $v$ it is known (and is straightforward to verify) that

$$
\operatorname{diff} \mathcal{E}[v]:=\left.\frac{d \mathcal{E}}{d t}\right|_{t=0}=-\int_{\partial \mathcal{G}(0)} v \cdot \kappa d \mathcal{H}^{1}-\sum_{x \in \mathcal{J}(0)} v(x) \cdot b(x) .
$$

where $\kappa$ is the mean curvature vector of the grain boundary. The mean curvature evolution of the grain boundary network is defined as the gradient flow of energy $\mathcal{E}$ with respect to the metric $g$, defined in (3). That is, the normal velocity $v$ by which the network moves is the minimizer of the Rayleigh functional:

$$
v \in \operatorname{argmin}_{w \in \mathcal{V}} \frac{1}{2} g(w, w)+\operatorname{diff} \mathcal{E}[w] .
$$


We note that if $b(x) \neq 0$ at any junction $x \in \mathcal{J}$, then the Rayleigh functional does not have a minimizer, since by taking $w$ to be larger and more localized around $x$, one can make the functional arbitrarily small. In other words, if $b(x) \neq 0$ the junction moves at infinite speed at that particular time so that for $t>0, b(x(t))$ (here, $x(t)$ is the time evolution of the particular junction) must be zero. Thus, the gradient flow takes place on the set of networks for which

$$
b(x)=0 \quad \text { for all } x \in \mathcal{J} .
$$

In such cases the minimizer of the Rayleigh functional is

$$
v=\kappa .
$$

We note that if more than three boundaries meet at a junction then there is direction which decreases the energy and splits the junction into several triple junctions, as proven by Cahn [6]. Thus the grain boundary networks have only triple junctions for all but finitely many times. The condition (4) is equivalent to the condition that all three angles at the junction are $120^{\circ}$, which is known as the Herring angle condition [13].

The presentation here is similar to the approach of Kinderlehrer and Lin [17], and Kinderlehrer, Livshits, and Ta'asan [18], though here we consider only the isotropic mean-curvature flow. On the other hand we indicate in more detail that the Herring condition at triple junctions follows from the gradient flow structure.

\section{Mean-Curvature Flow For Voronoi diagrams}

We consider a simplified model of the evolution of grain boundary networks by mean curvature. We restrict the configuration space from all grain boundary networks to ones which are Voronoi diagrams. The energy is still the total length of boundaries (2) and the inner product is still the integral over the boundaries of the dot product of normal velocities (3). In the regular case the network is described by the position of the centers of the Voronoi cells. For clarity, we first consider this case and furthermore assume that all junctions are triple junctions.

Specifically, the set of Voronoi diagrams with $N$ distinct centers is described as

$$
\mathcal{M}=\left\{\left(x_{1}, \ldots, x_{N}\right) \in\left(\mathbb{T}_{2}\right)^{N}: x_{i} \neq x_{j} \text { if } i \neq j\right\} / \sim
$$

where $\sim$ is the equivalence relation on $\left(\mathbb{T}_{2}\right)^{N}$ describing the relabeling of points. That is, $\left(x_{1}, \ldots, x_{N}\right) \sim\left(y_{1}, \ldots, y_{N}\right)$ if there exists a permutation $\sigma$ of $\{1, \ldots, N\}$ such that $y_{i}=\sigma\left(x_{i}\right)$ for all $i=1, \ldots, N$.

We begin to define the gradient flow on the configuration space of Voronoi diagrams: Let $\mathcal{E}(X)$ be the energy (the total perimeter). A tangent vector is given as a vector of velocities of the centers of Voronoi cells: $\delta x \in \mathbb{R}^{2 N}$. The metric is, as before, given by integrating over grain boundaries the square of the resulting normal velocity of the boundaries. It can be described by a quadratic, symmetric, positive definite matrix $Q$ :

$$
g(\delta x, \delta x)=\delta x^{T} Q \delta x .
$$

The gradient vector is given by

$$
\operatorname{grad} \mathcal{E}=Q^{-1}\left(D_{x} \mathcal{E}\right)^{T}
$$

The gradient flow is given by

$$
\dot{X}=-\operatorname{grad} \mathcal{E}(X) .
$$


To compute $\operatorname{grad} \mathcal{E}(X)$, we observe that it is natural to compute the energy (total perimeter) not directly in terms of the centers; but rather, in terms of the Voronoi diagram determined by these centers. We call line segments common to two cells edges, while the points belonging to three (or more) cells are called vertices (these are analogous to junctions in the general grain boundary networks). We denote the vertex that belongs to boundaries of cells with centers $x_{i}, x_{j}$, and $x_{k}$ by $v_{i j k}$. Let $V$ be the $m$-tuple of vertices. Define $F$ to be the function that assigns the vertices to configurations of centers:

$$
V=F(X),
$$

and let $P$ denote the derivative of $F$ :

$$
P=D F(X) .
$$

Define $\tilde{\mathcal{E}}(V)$ to be the perimeter expressed as the function of vertices. Then $\mathcal{E}(X)=$ $\tilde{\mathcal{E}}(F(X))$. Note that perturbation of centers induces a perturbation of vertices via $P$ :

$$
\delta v=P \delta x .
$$

Furthermore, note that the manifold of Voronoi diagrams is a submanifold of all grain boundary networks with straight edges. The metric on the larger manifold is described by

$$
\tilde{g}(\delta v, \delta v)=\delta v^{T} \tilde{Q} \delta v
$$

for $\tilde{Q}$ to be determined subsequently. The metric on the submanifold of Voronoi diagrams is the inherited one and thus

$$
g(\delta x, \delta x)=\tilde{g}(P \delta x, P \delta x)
$$

Hence

$$
Q=P^{T} \tilde{Q} P
$$

and the gradient vector is then expressible as

$$
\operatorname{grad} \mathcal{E}=Q^{-1}\left(D_{x} \mathcal{E}\right)^{T}=\left(P^{T} \tilde{Q} P\right)^{-1} P^{T}\left(D_{v} \tilde{\mathcal{E}}\right)^{T} .
$$

We next determine the explicit forms of the terms arising in the right-hand side of (10).

3.1. Expressions for gradient flow. In order to make use of (10), we must provide a prescription for computing $D_{v} \tilde{\mathcal{E}}, P$, and $\tilde{Q}$. Let $\delta v \in \mathbb{R}^{2 N}$. A straightforward computation reveals that the directional derivative of the function $\tilde{\mathcal{E}}$ in the direction $\delta v$ is

$$
D_{v} \tilde{\mathcal{E}} \cdot \delta v=\sum_{\alpha \in V} \sum_{\{\beta: \beta \in V,(\alpha, \beta) \in E\}} \frac{v_{\beta}-v_{\alpha}}{\left|v_{\beta}-v_{\alpha}\right|} \delta v_{\alpha},
$$

where $E=E(V)$ is the set of edges present in the Voronoi diagram with vertices $V$.

Next, we turn our attention to $\tilde{Q}$. The metric, which we introduced in (3), encodes the dissipation mechanism. It is equal to the integral of the squared normal velocity along the grain boundary network;

$$
\begin{aligned}
\tilde{g}(\delta v, \delta v)=\sum_{(\alpha, \beta) \in E} \frac{1}{3} \frac{1}{\left|v_{\alpha}-v_{\beta}\right|}\left(\left(\delta v_{\alpha} \cdot\left(v_{\alpha}-v_{\beta}\right)^{\perp}\right)^{2}\right. \\
\left.+\left(\delta v_{\alpha} \cdot\left(v_{\alpha}-v_{\beta}\right)^{\perp}\right)\left(\delta v_{\beta} \cdot\left(v_{\alpha}-v_{\beta}\right)^{\perp}\right)+\left(\delta v_{\beta} \cdot\left(v_{\alpha}-v_{\beta}\right)^{\perp}\right)^{2}\right)
\end{aligned}
$$


where we define

$$
R=\left(\begin{array}{rr}
0 & -1 \\
1 & 0
\end{array}\right)
$$

to be the matrix giving counterclockwise rotation of $90^{\circ}$, and define $v^{\perp}=R v$. Thus $\tilde{Q}$ is a block matrix with entries at the $2 \times 2$ block corresponding to the vertices $\alpha$ and $\beta$ given by

$$
\begin{cases}\frac{1}{3} \frac{1}{\left|v_{\alpha}-b_{\beta}\right|}\left(v_{\alpha}-v_{\beta}\right)^{\perp} \otimes\left(v_{\alpha}-v_{\beta}\right)^{\perp} & \text { if } \alpha \neq \beta \text { and }(\alpha, \beta) \text { is an edge, } \\ \frac{1}{3} \sum_{\{\tilde{\beta}:(\alpha, \tilde{\beta}) \text { is an edge }\}} \frac{1}{\left|v_{\alpha}-v_{\tilde{\beta}}\right|}\left(v_{\alpha}-v_{\tilde{\beta}}\right)^{\perp} \otimes\left(v_{\alpha}-v_{\tilde{\beta}}\right)^{\perp} & \text { if } \alpha=\beta, \\ 0 & \text { otherwise. }\end{cases}
$$

Finally, we need to describe the matrix $P$, the block matrix with entries $D_{l} v_{i j k}$. Let $v=v_{i j k}$ be the vertex for grains with centers $x_{i}, x_{j}$, and $x_{k}$. Note that

$$
\left|v-x_{i}\right|=\left|v-x_{j}\right|=\left|v-x_{k}\right|
$$

which is enough to describe $v$ as a function of $x_{i}, x_{j}, x_{k}$. We define increment and decrement operators on the indices $i, j, k$ so that $i^{+}=j, i^{-}=k$, and so on. Furthermore, we let $\sum_{h}$ stand for $\sum_{h \in\{i, j, k\}}$. Then the expression for the position of a vertex $v_{i j k}$ in terms of the centers $x_{i}, x_{j}$, and $x_{k}$ is:

$$
v_{i j k}=\frac{\sum_{h}\left(x_{h^{-}}^{T} x_{h^{-}}-x_{h^{+}}^{T} x_{h^{+}}\right) x_{h}^{\perp}}{2 \sum_{h} x_{h}^{T} x_{h^{+}}^{\perp}} .
$$

We note that if $x_{i}, x_{j}$, and $x_{k}$ were collinear then the denominator would be zero. However $x_{i}, x_{j}$ and $x_{k}$ cannot be collinear, since then the boundaries of cells would not intersect and hence $v_{i j k}$ would not exist. The fact that $x_{i}, x_{j}$, and $x_{k}$ are not collinear implies that the denominator is not zero. A simple way to show it is to note that the denominator is invariant under translation all points by the same vector. Thus we can assume $x_{i}=0$, in which case the claim is trivial to verify. For the convenience of the reader, a derivation of (13) is presented in Appendix A.

Differentiating (13) with respect to $x_{j}$, we obtain

$$
\frac{\partial v_{i j k}}{\partial x_{j}}=\frac{\left(x_{i}^{T} x_{i}-x_{k}^{T} x_{k}\right) R+2\left(x_{k}-x_{i}\right)^{\perp} x_{j}^{T}}{2 \sum_{h} x_{h}^{T} x_{h^{+}}^{\perp}}-\frac{\left(\sum_{h}\left(x_{h^{-}}^{T} x_{h^{-}}-x_{h^{+}}^{T} x_{h^{+}}\right) x_{h}^{\perp}\right)\left(\left(x_{k}-x_{i}\right)^{\perp}\right)^{T}}{2\left(\sum_{h} x_{h}^{T} x_{h^{+}}^{\perp}\right)^{2}} .
$$

Analogous expressions for derivatives with respect to $x_{i}$ and $x_{k}$ are obtained by applying the decrement and increment operators to (14). Combined with (10) they fully describe the gradient flow as long as points $x_{i}$ are distinct and as long as each vertex is at the boundary of exactly three Voronoi cells.

Additional complications arise when the Voronoi centers collide, and also when one vertex belongs to four or more Voronoi cells. To properly deal with the collisions we extend the configuration space. It turns out that the natural setting for the configuration space is a manifold with corners. Thus in the next section we briefly review the differential geometry of manifolds with corners. We subsequently return to the specific discussion of this complications for the Voronoi-constrained curvature flow. 


\section{Manifolds With CORners}

We first recall notions of the differential geometry of manifolds with corners, following the work of Joyce [16]. For $0 \leq k \leq d$, let $\mathbb{R}_{k}^{d}=[0, \infty)^{k} \times \mathbb{R}^{d-k}$. The only difference between the definition of a manifold and a manifold with corners is that a manifold with corners can have coordinate charts in $\mathbb{R}_{k}^{d}$ instead of $\mathbb{R}^{d}$. We note that $k$ is not fixed and that charts at different points of $\mathcal{M}$ can map to $\mathbb{R}_{k}^{d}$ with different values of $k$. A point $x \in \mathcal{M}$ is an interior point if there exists a chart into $\mathbb{R}^{d}$, it is a boundary point if it is not an interior point and there exists a chart into $[0, \infty) \times \mathbb{R}^{d-1}$, and a corner point otherwise.

Then for $x \in \mathcal{M}$ the tangent space is defined to be a vector space, even at boundary and corner points. At $x \in \mathcal{M}$ the inward sector, $I S_{x}$, is defined as the subset of the tangent space isomorphic to the equivalence classes of smooth curves $\gamma:[0, \varepsilon) \rightarrow \mathcal{M}$ such that $\gamma(0)=x$. We note that $I S$ is closed, and, since a convex combination of curves in $\mathbb{R}_{k}^{d}$ is a curve in $\mathbb{R}_{k}^{d}$, the set $I S_{x}$ is a convex cone for all $x \in \mathcal{M}$. We also note that the tangent space, $T_{x} \mathcal{M}$, is spanned by the vectors in $I S_{x}$.

4.1. Gradient flow on a manifold with corners. Given a smooth energy $\mathcal{E}: \mathcal{M} \rightarrow \mathbb{R}$ we would like to consider the gradient descent on $(\mathcal{M}, g)$, where $g$ is a metric on $\mathcal{M}$ defined in the standard way as a positive definite quadratic form on $T_{x} \mathcal{M}$. If $\mathcal{M}$ is a manifold without boundary and corners then the negative gradient vector of $\mathcal{E}$ at $x$ is defined by the requirement that

$$
g(-\operatorname{grad} \mathcal{E}, v)=-\operatorname{diff} \mathcal{E}[v] \quad \text { for all } v \in T_{x} \mathcal{M} .
$$

The vector $-\operatorname{grad}_{x} \mathcal{E}$ can also be identified as the minimizer of the Rayleigh functional:

$$
\operatorname{diff} \mathcal{E}[v]+\frac{1}{2} g(v, v) \quad \text { over all } v \in T_{x} \mathcal{M}
$$

We note that the above notions can be defined on a manifold with corners as well. However, the gradient flow $\dot{x}=-\operatorname{grad}_{x} \mathcal{E}$ would not have a solution in general, since at the boundary the negative gradient vector can point "outside" the manifold. We define the constrained negative gradient of $\mathcal{E}$ on the manifold with corners, $-\operatorname{cgrad}_{x} \mathcal{E}$, to be the minimizer of

$$
\operatorname{diff} \mathcal{E}[v]+\frac{1}{2} g(v, v) \quad \text { over all } v \in I S_{x} .
$$

Since $I S$ is closed and convex, and the functional minimized is convex, a unique minimizer exists.

We claim that the minimizer is nothing but the projection of $-\operatorname{grad} \mathcal{E}$ onto $I S_{x}$, that is, for all $v \in I S_{x}$

$$
g(v+\operatorname{grad} \mathcal{E}, v+\operatorname{grad} \mathcal{E}) \geq g(-\operatorname{cgrad} \mathcal{E}+\operatorname{grad} \mathcal{E},-\operatorname{cgrad} \mathcal{E}+\operatorname{grad} \mathcal{E}) .
$$

To prove this claim, note that by definition of $-\operatorname{cgrad} \mathcal{E}$ and $\operatorname{grad} \mathcal{E}$,

$$
g(\operatorname{grad} \mathcal{E}, v)+\frac{1}{2} g(v, v) \geq g(\operatorname{grad} \mathcal{E},-\operatorname{cgrad} \mathcal{E})+\frac{1}{2} g(-\operatorname{cgrad} \mathcal{E},-\operatorname{cgrad} \mathcal{E}),
$$

from which the claim follows by adding $g(\operatorname{grad} \mathcal{E}, \operatorname{grad} \mathcal{E})$ to both sides, doubling, and factoring.

We furthermore claim that

$$
g(\operatorname{cgrad} \mathcal{E}, \operatorname{grad} \mathcal{E}-\operatorname{cgrad} \mathcal{E})=0 .
$$




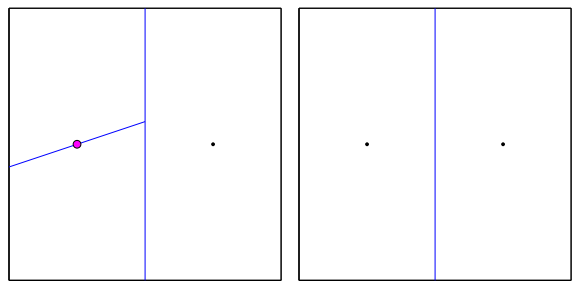

FiguRE 1. The Voronoi diagram for the points $x_{1}(0)=e_{1}, x_{2}(0)=$ $x_{3}(0)=-e_{1}$ (left, collided center marked with magenta circle) does not agree with the Voronoi diagram for the limiting configuration $x_{1}(0)=e_{1}$, $x_{2}(0)=-e_{1}$ (right). Here, $e_{1}:=(1,0)^{T}$.

The reason is that $I S$ is a cone, namely if $v \in I S$ then $s v \in I S$ for all $s \geq 0$. To show (17) we only need to consider the case $-\operatorname{cgrad} \mathcal{E} \neq 0$. By (16) we know that $s \mapsto g(-\operatorname{grad} \mathcal{E}+s \operatorname{cgrad} \mathcal{E},-\operatorname{grad} \mathcal{E}+s \operatorname{cgrad} \mathcal{E})$ is minimized when $s=1$. Thus, the derivative in $s$ at $s=1$ is zero, giving (17).

We define the gradient flow (descent) of the energy $\mathcal{E}$ on the manifold with corners as the solution of

$$
\dot{x}=-\operatorname{cgrad} \mathcal{E}(x) .
$$

Lemma 1. Let $x(t)$ be the gradient flow of a $C^{1}$ energy $\mathcal{E}$ on the manifold with corners $(\mathcal{M}, g)$. Then

$$
\frac{d \mathcal{E}(x(t))}{d t}=-g(\operatorname{cgrad} \mathcal{E}, \operatorname{cgrad} \mathcal{E})
$$

Proof. Using the definitions of $\operatorname{grad} \mathcal{E}$, gradient flow of $\mathcal{E}$ and (17)

$$
\frac{d \mathcal{E}(x(t))}{d t}=g(\operatorname{grad} \mathcal{E}, \dot{x})=-g(\operatorname{grad} \mathcal{E}, \operatorname{cgrad} \mathcal{E})=-g(\operatorname{cgrad} \mathcal{E}, \operatorname{cgrad} \mathcal{E})
$$

\section{Collision of Centers and topological Changes}

Now we return to the discussion of the major complicating factors in the evolution: collisions of centers and changes in the topology of the Voronoi diagram. These complications can occur separately or simultaneously. The collisions of centers in particular raise new issues. To illustrate this consider a curve in $\left(\mathbb{T}_{2}\right)^{3}$ given by $\left(x_{1}(t), x_{2}(t), x_{3}(t)\right)$. Assume that for $t<0$ the points are distinct, but that for $t=0, x_{1}(0) \neq x_{2}(0)=x_{3}(0)$. The Voronoi diagrams with centers $\left(x_{1}(t), x_{2}(t), x_{3}(t)\right)$ do not converge to the diagram with centers $\left(x_{1}(0), x_{2}(0)\right)$ at $t \rightarrow 0-$. See Figure 1 for a simple example. Thus considering the set of $n$-tuples of possibly nondistinct points in $\mathbb{T}_{2}$ is not the correct way to compactify $\mathcal{M}$ in general. Another way to see this is to notice that when when centers are sufficiently close, a small change in the location of centers (particularly, the angle between them) can lead to (arbitrarily) fast motion of boundaries.

To be precise, we consider the three possible scenarios:

- Pure boundary event. An example is depicted in Figure 2(a). The limiting Voronoi diagram is not the Voronoi diagram for the collided centers. A pure boundary event always occurs when exactly two centers collide, but may happen for the collision of any number of centers. The name "boundary event" is chosen 


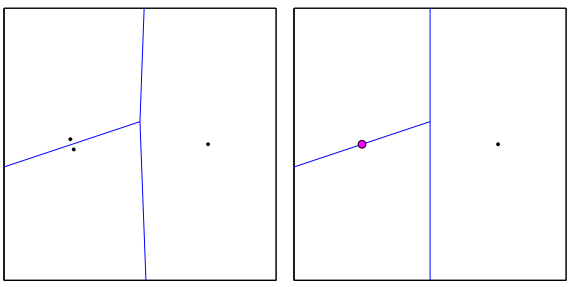

(a)
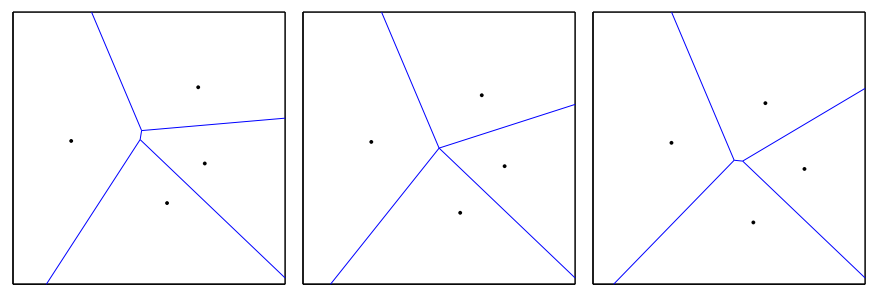

(b)
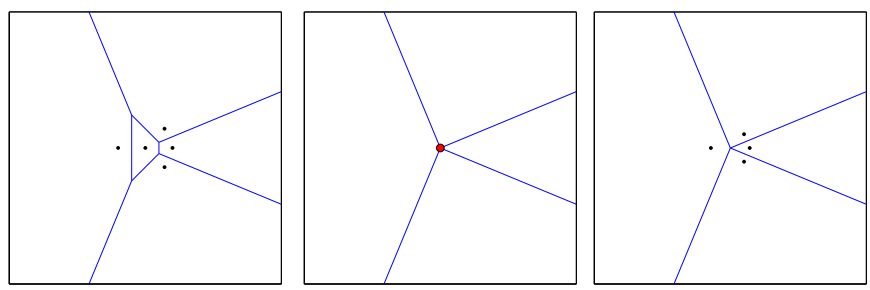

(c)

Figure 2. (a) A boundary event occurs when two or more centers collide. (b) During a change in topology, a vertex instantaneously belongs to four Voronoi cells. (c) The disappearance of the central grain causes the creation of a vertex belonging to four Voronoi cells corresponding to the new collided center. This center typically breaks up following the grain disappearance.

to indicate that to handle this case we need to extend $\mathcal{M}$ to a manifold with boundary (in fact with corners too). This case is handled in Section 5.1.

- Pure topological change. It may also happen during the evolution that a vertex belongs to four or more Voronoi cells. This typically happens during changes of the topology of the Voronoi diagram; namely, when the neighborhood structure of a cell changes. See Figure 2(b). This possibility is handled in Section 5.2.

- Boundary event with topological change. A grain disappearance event combines a boundary event with a change in the topology of the Voronoi diagram. The disappearance event is driven by the collision of centers of the disappearing grain and all its neighbors. The collision event removes the Voronoi cell of the central grain, as its area goes to zero, and gives rise to a boundary event involving all the surrounding centers. Typically, the collided centers will again separate following the grain disappearance. See Figure 2(c).

5.1. Collisions of centers. Returning to the model at hand, the issue is how to identify the set of all possible limits of Voronoi diagrams during a boundary event and assign it 
the appropriate topology. In other words, the task is to find the correct compactification of $\mathcal{M}$. This problem was carefully considered by Lindenbergh in his doctoral thesis [22] and together with van der Kallen and Siersma [21]. The way the manifold is compactified is by introducing the set of directed angles between centers. Namely let $\mathcal{P}=\left(\mathbb{T}_{2}^{N} \times\left(S^{1}\right)^{N(N-1))}\right) / \sim$ where the equivalence relation $\sim$ is defined by

$$
\left(\left(x_{i}\right)_{i=1, \ldots, N},\left(\alpha_{i, j}\right)_{i, j=1, \ldots, N, i \neq j}\right) \sim\left(\left(y_{i}\right)_{i=1, \ldots, N},\left(\beta_{i, j}\right)_{i, j=1, \ldots, N, i \neq j}\right)
$$

if there exists a permutation $\sigma$ such that $y_{i}=x_{\sigma(i)}$ and $\beta_{i, j}=\alpha_{\sigma(i), \sigma(j)}$. Consider the mapping

$$
\Psi: \mathcal{M} \rightarrow \mathcal{P}
$$

defined by

$$
\Psi\left(\left(x_{i}\right)_{i=1, \ldots, N}\right)=\left(x_{i}\right)_{i=1, \ldots, N} \times\left(\frac{x_{i}-x_{j}}{\left|x_{i}-x_{j}\right|}\right)_{i, j=1, \ldots, N, i \neq j} .
$$

In [22] the compactification $\overline{\mathcal{M}}$ of $\mathcal{M}$ was defined as the closure of $\Psi(\mathcal{M})$ in $\mathcal{P}$. It was shown that the continuous evolution of points in $\overline{\mathcal{M}}$ produces continuous motion of cell boundaries. It was furthermore remarked that $\overline{\mathcal{M}}$ is a manifold with corners. Here we provide a somewhat different, but equivalent description of $\overline{\mathcal{M}}$. We also provide explicit local coordinates for $\overline{\mathcal{M}}$ at the boundary and at the corners. Furthermore we express the metric in terms of these coordinates and use them to express and compute the gradient flow in the local coordinates.

Since the general coordinates are a bit involved we first present the cases when there can be only one collision of (two of more) centers. Since the local coordinates for two centers are particularly simple we present them first as a special case.

5.1.1. Local coordinates for 2 centers. These coordinates allow for the collisions of two centers. By relabeling we can assume that the centers which we allow to collide are the first two. Let $A=\left\{\bar{y}_{12}, \bar{y}_{3}, \ldots, \bar{y}_{N}\right\}$ be distinct points on $\mathbb{T}_{2}$. Let $\varepsilon=$ $\frac{1}{3} \min _{y, \tilde{y} \in A} \operatorname{dist}(y, \tilde{y})$. Let $I_{\theta}$ be an open interval of length less than $\pi$. We define local coordinates on $U=B\left(\bar{y}_{12}, \varepsilon\right) \times[0, \varepsilon) \times S^{1} \times \Pi_{i=3}^{N} B\left(\bar{y}_{i}, \varepsilon\right)$. While $U$ is not truly a subset of $\mathbb{R}^{2 N}$ since some of the coordinates are in $S^{1}$ and in $\mathbb{T}_{2}$ it is straightforward to provide local coordinates in $\mathbb{R}^{2 N}$ for $U$, so, for simplicity, we work directly with $U$.

We note that since $d \in[0, \varepsilon)$ the chart on $U$ is for a manifold with boundary. The coordinate change from $\stackrel{\circ}{U}=B\left(\bar{y}_{12}, \varepsilon\right) \times(0, \varepsilon) \times I_{\theta} \times \Pi_{i=3}^{N} B\left(\bar{y}_{i}, \varepsilon\right)$ to the standard coordinates is as follows: To $\left(z, d, f_{2}, y_{3}, \ldots, y_{N}\right) \in \stackrel{\circ}{U}$ we associate

$$
\begin{aligned}
& x_{1}=z+d f_{1} \\
& x_{2}=z+d f_{2} \\
& x_{i}=y_{i} \quad \text { for } i=3, \ldots, N,
\end{aligned}
$$

where $f_{1}=-f_{2}$. We note that the inverse of this transformation gives $z=\frac{1}{2}\left(x_{1}+x_{2}\right)$, $d=\left|x_{2}-z\right|$, and $f_{2}=\left(x_{2}-z\right) / d$.

The grain boundary network that corresponds to $\left(z, d, f_{2}, y_{3}, \ldots, y_{n}\right) \in U$ is as follows: If $d>0$, then it is just the Voronoi diagram corresponding to centers $x_{1}, \ldots, x_{N}$. If $d=0$, consider the Voronoi diagram corresponding to centers $x_{2}, \ldots, x_{N}$ and denote the cells by $\tilde{G}_{i}, i=2, \ldots, N$. We need to define the cells $G_{j}, j=1, \ldots, N$ of the full configuration. For $j=3, \ldots, N$ set $G_{j}=\tilde{G}_{j}$ and define

$$
G_{1}=\left\{w \in \tilde{G}_{2}: w \cdot f_{2}<0\right\} \text { and } G_{2}=\left\{w \in \tilde{G}_{2}: w \cdot f_{2}>0\right\} .
$$




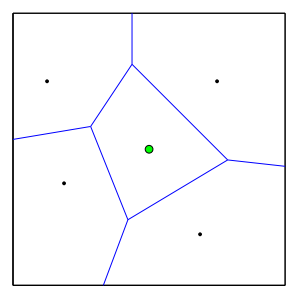

(a)

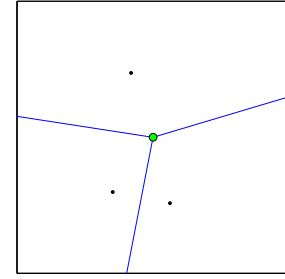

(b)

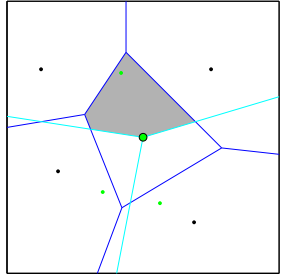

(c)

Figure 3. (a) The green center, $z$, has multiplicity 3. (b) The cells $S_{i}$ are depicted with the center $z$. (c) An exemplar $G_{i}=\tilde{G}_{1} \cap S_{i}$ is shaded in gray.

5.1.2. Local coordinates for $n>2$ centers. Again by relabeling we can assume that the first $n<N$ centers are allowed to collide. Let $A=\left\{\bar{y}_{c}, \bar{y}_{n+1}, \ldots, \bar{y}_{N}\right\}$ be a set of $N-n+1$ distinct points in $\mathbb{T}_{2}$. Let $\varepsilon=\frac{1}{3} \min _{y, \tilde{y} \in A} \operatorname{dist}(y, \tilde{y})$. Let $W_{f}=$ $\left\{\left(f_{2}, \ldots, f_{n}\right) \in \mathbb{R}^{2 n-2}:(\forall i=2, \ldots, n)(\forall j=i+1, \ldots, n)\left|f_{i}\right|>\frac{1}{2}\right.$ and $\left.\left|f_{i}-f_{j}\right|>\frac{1}{2}\right\}$ and $O_{d}=\left\{\left(f_{3}, \ldots, f_{n}\right) \in \mathbb{R}^{2(n-3)}:(\forall i=2, \ldots, n)\left|f_{i}\right|<\frac{1}{d}\right\}$. Let $U=B\left(\bar{y}_{c}, \varepsilon\right) \times$ $\left(\bigcup_{d \in[0, \varepsilon)}\{d\} \times\left(W_{f} \cap\left(S^{1} \times O_{d}\right)\right)\right) \times \Pi_{j=n+1}^{N} B\left(\bar{y}_{j}, \varepsilon\right)$. We note that $U$ is diffeomorphic to $[0,1) \times W$ where $W$ is a open subset of $\mathbb{R}^{2 N-1}$. The coordinate change from $\left(z, d, f_{2}, \ldots, f_{n}, y_{n+1}, \ldots, y_{N}\right)$ in the interior of $U$ to standard coordinates is

$$
\begin{aligned}
& x_{i}=z+d f_{i} \quad \text { for } i=1, \ldots, n \\
& x_{i}=y_{i} \quad \text { for } i=n+1, \ldots, N .
\end{aligned}
$$

where $f_{1}=-f_{2}$. We remark that the $f_{h} \in \mathbb{R}^{2}$ are nondimensional quantities. One should note that if $d=0$ then all of the first $n$ centers collide and if $d>0$ then there are no collisions of centers. Also since $d \in[0, \varepsilon)$ the above is a chart at the boundary of a manifold.

The grain boundary network which corresponds to $\left(z, d, f_{2}, \ldots, f_{n}, y_{n+1}, \ldots, y_{N}\right)$ is defined as follows. If $d>0$ then it is just the Voronoi diagram corresponding to $\left(x_{1}, \ldots, x_{N}\right)$. If $d=0$ then consider the Voronoi diagram corresponding to centers $z, x_{n+1}, \ldots, x_{N}$ and denote its cells by $\tilde{G}_{1}, \tilde{G}_{n+1}, \ldots, \tilde{G}_{N}$. Consider also the Voronoi diagram with centers $z+f_{1}, \ldots, z+f_{n}$, and denote its cells by $S_{i}$. The cells in the full configuration are given by

$$
\begin{aligned}
& G_{i}=\tilde{G}_{1} \cap S_{i} \quad \text { for } i=1, \ldots, n \\
& G_{i}=\tilde{G}_{i} \quad \text { for } i=n+1, \ldots, N
\end{aligned}
$$

as depicted on Figure 3(c).

5.1.3. General coordinates with more than one collision. Here we describe the local coordinates where $m_{1}, m_{2}, \ldots, m_{k}$ with $m_{j} \geq 2$ for $j=1, \ldots, k$ centers are allowed to collide. We can relabel the centers so that the first $m_{1}$ centers may be colliding together, the next $m_{2}$ colliding together, and so on. Let $m=m_{1}+\cdots+m_{k}$. Let $A=$ $\left\{\bar{y}_{c 1}, \ldots, \bar{y}_{c k}, \bar{y}_{m+1}, \ldots, \bar{y}_{N}\right\}$ be set of $k+N-m$ distinct points in $\mathbb{T}_{2}$ and let as before $\varepsilon=$ $\frac{1}{3} \min _{y, \tilde{y} \in A} \operatorname{dist}(y, \tilde{y})$. For each $j=1, \ldots, k$ we define coordinates $\left(z_{j}, d_{j}, f_{1, j}, \ldots, f_{m_{j}, j}\right)$ as for a single collision discussed in Section 5.1.2. The coordinates for each of the collision are concatenated and finally $N-m$ coordinates for the non-colliding centers 
are added on. We note that since $d_{j} \in[0, \varepsilon)$ then if $k \geq 2$ these local coordinates correspond to a coordinate chart at a corner.

5.1.4. Expressing Voronoi vertex locations in terms of collided centers. Here we investigate the correspondence $F$ which gives the coordinates of vertices corresponding to the configuration given in the new ("collided") coordinates. This correspondence is analogous to $F$ in (8). More precisely we find the derivative of this correspondence $P=D F$, as in (9). We first consider the case that each junction is an intersection of precisely three cells. Let $x_{i}, x_{j}$, and $x_{k}$ represent centers expressed in the local coordinates, (19), and $x_{\ell}$ and $x_{m}$ represent centers expressed in the standard (Euclidean) coordinates, (20). We use the notation we introduced in Subsection 3.1.

Vertices of the form $v_{i \ell m}$. Let $\alpha$ be one of the new coordinates $\left(z, d\right.$ or $\left.f_{s}\right)$. For vertices of the form $v_{i \ell m}$, we can simply use the chain rule to obtain

$$
\frac{\partial v_{i \ell m}}{\partial \alpha}=\frac{\partial v_{i \ell m}}{\partial x_{i}} \frac{\partial x_{i}}{\partial \alpha}
$$

where $\partial v_{i \ell m} / \partial x_{i}$ is computed by (14), and $\partial x_{i} / \partial_{\alpha}$ is easily read off from (19). Here, the application of the chain rule is numerically stable as $\partial v_{i \ell m} / \partial x_{i}$ is not large.

Vertices of the form $v_{i j \ell}$. In contrast to the previous case, if $d>0$ is small, $\partial v_{i j \ell} / \partial x_{i}$ is expected to be large due to the proximity of $x_{i}$ and $x_{j}$. To find the derivative in a way which is numerically stable and allows for $d=0$ we simplify the expression for $v_{i j \ell}$ in terms of the collapsed coordinates and take derivatives directly. The expression

$$
v_{i j \ell}=\frac{c_{1}\left(z-x_{\ell}\right)^{\perp}+c_{2} f_{i}^{\perp}+c_{3} f_{j}^{\perp}}{c_{0}}:=\frac{u}{c_{0}},
$$

where

$$
\begin{aligned}
& c_{1}=2 z \cdot\left(f_{i}-f_{j}\right)+d\left(f_{i} \cdot f_{i}-f_{j} \cdot f_{j}\right), \\
& c_{2}=x_{\ell} \cdot x_{\ell}-z \cdot z-2 d\left(z \cdot f_{j}\right)-d^{2}\left(f_{j} \cdot f_{j}\right), \\
& c_{3}=-x_{\ell} \cdot x_{\ell}+z \cdot z+2 d\left(z \cdot f_{i}\right)+d^{2}\left(f_{i} \cdot f_{i}\right), \\
& c_{0}=2\left(d\left(f_{i} \cdot f_{j}^{\perp}\right)+\left(f_{i}-f_{j}\right) \cdot\left(z-x_{\ell}\right)^{\perp}\right),
\end{aligned}
$$

is obtained from (13), with the collapsed coordinates (19) substituted in. When $d \neq 0$, $c_{0} \neq 0$ since $f_{i}, f_{j}, x_{\ell}-z$ are not collinear. If $d=0$ then the edge between cells described by $f_{i}$ and $f_{j}$ is orthogonal to $f_{i}-f_{j}$, so again $c_{0}=0$ if and only if $f_{i}, f_{j}$, and $x_{\ell}-z$ are collinear. 
We determine the derivatives of $v_{i j \ell}$ wrt. the new coordinates to be:

$$
\begin{aligned}
\frac{\partial v_{i j \ell}}{\partial z} & =\frac{2}{c_{0}}\left(-\frac{1}{c_{0}} u \otimes\left(f_{j}-f_{i}\right)^{\perp}+\left(z-x_{\ell}\right)^{\perp} \otimes\left(f_{i}-f_{j}\right)+\frac{c_{1}}{2} R-f_{i}^{\perp} \otimes x_{j}+f_{j}^{\perp} \otimes x_{i}\right), \\
\frac{\partial v_{i j \ell}}{\partial d} & =\frac{2}{c_{0}}\left(-\frac{f_{i} \cdot f_{j}^{\perp}}{c_{0}} u+\frac{1}{2}\left(f_{i} \cdot f_{i}-f_{j} \cdot f_{j}\right)\left(z-x_{\ell}\right)^{\perp}-\left(x_{j} \cdot f_{j}\right) f_{i}^{\perp}+\left(x_{i} \cdot f_{i}\right) f_{j}^{\perp}\right), \\
\frac{\partial v_{i j \ell}}{\partial f_{i}} & =\frac{2}{c_{0}}\left(-\frac{1}{c_{0}} u \otimes\left(x_{j}-x_{\ell}\right)^{\perp}+\left(x_{j}-x_{\ell}\right)^{\perp} \otimes x_{i}+\frac{c_{2}}{2} R\right), \\
\frac{\partial v_{i j \ell}}{\partial f_{j}} & =\frac{2}{c_{0}}\left(-\frac{1}{c_{0}} u \otimes\left(x_{\ell}-x_{i}\right)^{\perp}+\left(x_{\ell}-x_{i}\right)^{\perp} \otimes x_{j}+\frac{c_{3}}{2} R\right), \\
\frac{\partial v_{i j \ell}}{\partial x_{\ell}} & =\frac{2}{c_{0}}\left(-\frac{1}{c_{0}} u \otimes\left(f_{i}-f_{j}\right)^{\perp}-\frac{c_{1}}{2} R+\left(f_{i}-f_{j}\right)^{\perp} \otimes x_{\ell}\right) .
\end{aligned}
$$

Additional simplifications can be made in the $n=2$ case. Then $f_{i}=-f_{j}$, so

$$
v_{i j \ell}=\frac{2\left(z \cdot f_{i}\right)\left(z-x_{\ell}\right)^{\perp}+\left(x_{\ell} \cdot x_{\ell}-z \cdot z-d^{2}\right) f_{i}^{\perp}}{2 f_{i} \cdot\left(z-x_{\ell}\right)^{\perp}} .
$$

Vertices of the form $v_{i j k}$. With collided centers $x_{i}, x_{j}$ and $x_{k},(13)$ can be simplified. Using the coordinates $x_{i}=z+d f_{i}$ (i.e. $n \geq 3$ ), we obtain:

$$
v_{i j k}=z+d \frac{\sum_{h}\left(f_{h^{-}} \cdot f_{h^{-}}-f_{h^{+}} \cdot f_{h^{+}}\right) f_{h}^{\perp}}{2 \sum_{h} f_{h} \cdot f_{h^{+}}^{\perp}} .
$$

Observe that this expression is simply (13) translated into the frame of reference of the collapsed coordinates.

Differentiating this expression, we obtain:

$$
\begin{aligned}
\frac{\partial v_{i j k}}{\partial z} & =\mathbb{I}_{2} \\
\frac{\partial v_{i j k}}{\partial d} & =\frac{\sum_{h}\left(f_{h^{-}} \cdot f_{h^{-}}-f_{h^{+}} \cdot f_{h^{+}}\right) f_{h}^{\perp}}{2 \sum_{h \in\{i, j, k\}} f_{h} \cdot f_{h^{+}}^{\perp}} \\
\frac{\partial v_{i j k}}{\partial f_{j}} & =d\left(\frac{\left(f_{i} \cdot f_{i}-f_{k} \cdot f_{k}\right) R+2\left(f_{k}-f_{i}\right)^{\perp} \otimes f_{j}}{2 \sum_{h} f_{h} \cdot f_{h^{+}}^{\perp}}\right. \\
& \left.-\frac{\left(\sum_{h}\left(f_{h^{-}} \cdot f_{h^{-}}-f_{h^{+}} \cdot f_{h^{+}}\right) f_{h}^{\perp}\right) \otimes\left(f_{k}-f_{i}\right)^{\perp}}{2\left(\sum_{h} f_{h} \cdot f_{h^{+}}^{\perp}\right)^{2}}\right)
\end{aligned}
$$

and analogous expressions for the derivatives with respect to $f_{i}$ and $f_{k}$.

5.1.5. Gradient flow in the "collided" coordinates. The correspondence, above, between the collided coordinates and the vertices enables us to find the gradient of the energy in the collided coordinates. More precisely, above we computed the coefficients of the matrix $P$ of derivatives of the location of vertices with respect to collided coordinates. The gradient vector is then defined by (10) where $\tilde{\mathcal{E}}$ and $\tilde{Q}$ are given by (11) and (12), as before. Finding the negative constrained gradient vector $-\operatorname{cgrad} \mathcal{E}$ is then straightforward. If the $d$ coordinate of the configuration in collided coordinates is positive then $-\operatorname{cgrad} \mathcal{E}=-\operatorname{grad} \mathcal{E}$. If $d=0$ then as we showed in Section 4 , the vector $-\operatorname{cgrad} \mathcal{E}$ is the projection of $-\operatorname{grad} \mathcal{E}$ to the inward sector $I S$. The inward sector $I S$ of the configuration is the set of tangent vectors whose $d$-coordinate is positive. So if the $d$-coordinate 
of $-\operatorname{grad} \mathcal{E}$ is nonnegative then $-\operatorname{cgrad} \mathcal{E}=-\operatorname{grad} \mathcal{E}$; otherwise $-\operatorname{cgrad} \mathcal{E}$ is obtained by changing the $d$ coordinate of $-\operatorname{grad} \mathcal{E}$ to 0 .

5.2. High-order vertices. It may happen during the evolution that a vertex belongs to four or more Voronoi cells. This typically happens during changes of the topology of the network; namely, when the neighborhood structure of a cell changes or when a cell disappears. See Figure 2. While the formulae for the vertex locations derived in the Appendix A still hold at the given time, if a topological change occurs the vertex may no longer be on the boundary of given centers for future times. Thus the gradient computed under one assumption on the network is not valid if the future network is not as assumed. Furthermore the energy is not a differentiable function of center positions at the times of topological events. The way we resolve that is as follows. For each inward tangent vector, corresponding to an infinitesimal perturbation of the centers, the network develops a particular topology for small future times. This divides the inward sector of the tangent space into cones of directions that lead to a particular network topology. Under each geometric assumption we compute the steepest descent vector within the closure of the cone, that is, the minimizer of the Rayleigh functional (15) over the cone. If the minimizer exists (since some of the cones are not closed there may be no minimizer) we call it an admissible vector. Then we set the gradient vector to be the admissible vector which decreases the energy the most. We show that at least one of the cones is closed and thus the Rayleigh functional has a minimizer over this particular cone. So at least one admissible vector exists.

Let us first present the details in the quadruple vertex case. We denote by $v_{i j k \ell}$ a vertex for cells with centers $x_{i}, x_{j}, x_{k}$, and $x_{\ell}$, such that the centers $x_{i}$ and $x_{k}$ do not share an edge meeting this vertex. The issue is that small variations in the positions of the centers can lead to different network topology. For example, the network can have the topology where $v_{i j k \ell}$ splits into $v_{i j k}$ and $v_{i k \ell}$ (case 1), where it splits into $v_{i j \ell}$ and $v_{j k \ell}$ (case 2), and where $v_{i j k l}$ remains at the boundary of four cells (case 3 ).

Define $\phi: X \rightarrow \mathcal{G}$ to be the mapping that assigns the grain boundary network to the centers. Let $C_{q}=\{\delta x \in I S: \exists \varepsilon>0$ s.t. $\phi(x+h \delta x)$ has the topology of case $q, \forall h \in$ $(0, \varepsilon)\}$ for $q=1,2,3$. We note that each of the sets is a cone and that $C_{1}$ and $C_{2}$ are open cones while $C_{3}$ is a closed cone. Furthermore $C_{3}=\bar{C}_{1} \cap \bar{C}_{2}$. The gradient vector is defined as follows. For $q=1,2,3$, find the minimizer of the Rayleigh functional over $\bar{C}_{q}$. It can be shown (see Section 4 for details) that the minimizer is the projection of $\operatorname{grad}_{q} \mathcal{E}$, the gradient vector defined by (10) under the topology of case $q$, to the cone $\bar{C}_{q}$. If the minimizer belongs to $C_{q}$ itself it is deemed admissible. We note that since $C_{3}$ is closed there is always at least one admissible vector. We define the gradient vector to be the admissible vector which decreases the energy the most. That is the admissible vector $v$ for which $-\operatorname{diff} \mathcal{E}[v]$ is the largest (remembering that $\operatorname{diff} \mathcal{E}$ is computed for each $v$ under the assumption of the appropriate network topology). As in Lemma 1 one can show that the final gradient vector is the longest among the admissible vectors. If it happens that there are two admissible vectors of the same length, then any one of them can be chosen (and thus one cannot expect uniqueness of the evolution in general). An example where this happens is when $x_{i}, x_{j}, x_{k}$, and $x_{\ell}$ are vertices of a square. We remark that our results on the upper bound on the rate of coarsening do not depend on which admissible vector is chosen. We also note that generically the quadruple vertices are energetically unstable and instantaneously break up. 
Before we define the evolution at the higher-order vertices, we remark that they too are unstable. The approach to defining gradient flow is analogous to one for quadruple vertices. For each possible local geometry that may arise there exists a cone of inward tangent directions that take the dynamics into the given geometry. For regular geometries (ones where all vertices are triple), the cones are open. The cones corresponding to cases when some of the vertices are quadruple or of higher multiplicity have empty interior and the one that preserves the multiplicity of the junction is closed. Again for each possible geometry a "gradient" vector $\operatorname{grad} \mathcal{E}$ is computed using (10). As before, the projection of the vector $-\operatorname{grad} \mathcal{E}$ to the closure of the cone is the minimizer of the Rayleigh functional (15) over the closure of the cone. If this projection belongs to the cone itself then it is an admissible vector. Since at least one of the cones is closed there always exists an admissible vector. The negative constrained gradient vector is defined to be the admissible vector which decreases the energy the most. As before it turns out to be the longest of the admissible vectors.

Finally, if there is more than one junction of multiplicity at least four at the same time the discussion is the same as above. The inward sector of the tangent space is still divided into cones, etc. The only difference is that the combinatorics of the possible geometries becomes quite complicated. One needs to consider all local geometries at each cone simultaneously, so the number of possible cases (and thus cones) is in most cases the product of the number of cones at each of the vertices.

\section{Coarsening Rate}

We consider the gradient flow of Voronoi diagrams on torus $\mathbb{T}_{2}=[0, \Lambda]^{2}$ and define $\overline{\mathcal{E}}=\mathcal{E} / \Lambda^{2}$ to be the energy density. We use the framework of Kohn and Otto [20] to show a universal upper bound on the rate of coarsening of the evolution of Voronoi diagrams by mean curvature. The framework uses an additional quantity $L$ associated to a configuration and proving two inequalities that relate $\mathcal{E}$ and $L$. An ODE argument is then used to obtain a time-averaged lower bound on the energy, which provides an upper bound on the rate of coarsening. For the mean-curvature flow natural interpolation inequality would be of the form $\overline{\mathcal{E}} L^{2} \gtrsim 1$ and a natural dissipation inequality would be $-\frac{d \overline{\mathcal{E}}}{d t} \geq\left(\frac{d L}{d t}\right)^{2}$. If they held they would imply a weak form of $\overline{\mathcal{E}} \gtrsim t^{-1 / 2}$ as desired. As discussed in [27], when the coarsening system has a gradient flow structure there is a natural choice of $L$ : set $L$ to be the distance in the configuration space, with respect to metric of the gradient flow, to a fixed configuration. However the metric on the set of grain boundary networks with respect to which the mean-curvature flow is a gradient flow, namely the $L^{2}$ inner product of velocities (3), is degenerate in the sense that the distance between any two networks is zero. This follows from work of Mumford and Michor [24].

To overcome that, we use the length of the solution curve, instead of using the distance to a fixed configuration, to define $L$. This is made precise in (23) below. In this way $L$ depends not only on the given grain boundary network, but rather on its history during the gradient flow. We note that a consequence of the definition is that the dissipation inequality becomes an equality. 
We now provide the details of the approach and its proof. Using the energy defined in (2) we define the characteristic length to be

$$
\ell=\frac{1}{\overline{\mathcal{E}}}:=\frac{\Lambda^{2}}{\mathcal{E}}
$$

The quantity $\ell$ suggests what is the typical one-dimensional size of grains. First, we show that only small proportion of the total area can be in grains with area much less than $\ell^{2}$.

Lemma 2. Given a grain boundary network $\mathcal{G}=\left\{G_{1}, \ldots, G_{N}\right\}$ let $\ell$ be defined by (21). Consider $\delta \in(0,1)$. Let $I_{\text {small }}=\left\{i \in\{1, \ldots, N\}:\left|G_{i}\right|<\delta \ell^{2}\right\}$. Then

$$
\frac{1}{\Lambda^{2}} \sum_{i \in I_{\text {small }}}\left|G_{i}\right|<\sqrt{\frac{\delta}{\pi}} .
$$

We remark that while we only state the lemma in 2D, analogous statements hold in any dimension, and their proof relies on isoperimetric inequality, as does the one below.

Proof. Using the isoperimetric inequality, which states that $4 \pi|G| \leq \operatorname{Per}(G)^{2}$, we obtain

$$
\begin{aligned}
\frac{1}{\ell}=\overline{\mathcal{E}} & \geq \frac{1}{2 \Lambda^{2}} \sum_{i \in I_{\text {small }}} \operatorname{Per}\left(G_{i}\right) \\
& \geq \frac{\sqrt{\pi}}{\Lambda^{2}} \sum_{i \in I_{\text {small }}} \sqrt{\left|G_{i}\right|}=\frac{\sqrt{\pi}}{\Lambda^{2}} \sum_{i \in I_{\text {small }}} \frac{\left|G_{i}\right|}{\sqrt{\left|G_{i}\right|}} \\
& \geq \frac{\sqrt{\pi}}{\Lambda^{2}} \sum_{i \in I_{\text {small }}} \frac{\left|G_{i}\right|}{\sqrt{\delta} \ell} .
\end{aligned}
$$

Therefore

$$
\sqrt{\frac{\delta}{\pi}} \geq \frac{1}{\Lambda^{2}} \sum_{i \in I_{\text {small }}}\left|G_{i}\right| .
$$

We recall that the first variation of volume gives that if $\left\{G_{t}\right\}_{t}$ is family of bounded sets with $C^{1}$ boundary moving by a continuous normal velocity $v$, then

$$
\frac{d\left|G_{t}\right|}{d t}=\int_{\partial G_{t}} v \cdot \nu d \mathcal{H}^{1}\left\llcorner\partial G_{t},\right.
$$

where $\nu$ is the unit outward normal vector. Using a straightforward smoothing argument the regularity requirements above can be relaxed to continuous and piecewise $C^{1}$ boundaries moving continuously on the $C^{1}$ parts. Let $A$ be an open set with with piecewise $C^{1}$ boundary. Applying the above to $A \cap G_{t}$ we obtain that

$$
\left|G_{t_{2}} \cap A\right|-\left|G_{t_{1}} \cap A\right| \leq \int_{t_{1}}^{t_{2}} \int_{\left(\partial G_{t}\right) \cap A}|v| d \mathcal{H}^{1}\left\llcorner\left(\partial G_{t}\right) \cap A d t\right.
$$

Theorem 3. Let $\mathcal{G}_{t}, t \geq 0$ be a continuous evolution of a grain boundary network such that at each time each grain is convex. The grains can disappear during the evolution 
but cannot reappear and no new grains are created. Assume that at $t=0$ and for all $i=1, \ldots, N,\left|G_{i}\right| \leq 1$. Let $v$ be the normal velocity of the grain boundaries. Let

$$
L(t)=1+\frac{1}{\Lambda} \int_{0}^{t} \sqrt{\int_{\partial \mathcal{G}_{s}} v(x, s)^{2} d \mathcal{H}^{1}\left\llcorner_{\partial \mathcal{G}_{s}}(x)\right.} d s .
$$

Then, for all $t \geq 0$

$$
\overline{\mathcal{E}} L^{2} \geq \frac{1}{32}
$$

We remark that while we only consider this theorem in two dimensions it actually holds in any dimension (with the constant on the right hand side depending on the dimension).

Proof. If $\overline{\mathcal{E}}(t) \geq \frac{1}{2}$ the inequality is trivial, as $L \geq 1$. Let us consider the case $\overline{\mathcal{E}}(t)<\frac{1}{2}$. Take $\delta=2 / \ell^{2}$. From Lemma 2 it follows that $\frac{1}{\Lambda^{2}} \sum_{I:\left|G_{i}(t)\right|<2}\left|G_{i}(t)\right|<\frac{1}{2}$. Let $J=\{i$ : $\left.\left|G_{i}(t)\right| \geq 2\right\}$. Then

$$
\frac{1}{\Lambda^{2}} \sum_{i \in J}\left|G_{i}(t)\right| \geq \frac{1}{2}
$$

that is, at least half of the total area lies in grains of area two or more.

It is well known that convex subsets of any set have lesser perimeter than the set itself. In particular Cauchy's surface-area formula (see Sec 5.5 in [19]) can be seen as a quantitative form of this statement. Thus

$$
\operatorname{Per}\left(G_{i}(s) \cap G_{i}(t)\right) \leq \operatorname{Per}\left(G_{i}(t)\right) \quad \text { for all } s \leq t
$$

To prove the interpolation inequality we estimate the dissipation only along the evolution of the grains which are of area at least 2 to time $t$, namely grains $G_{i}(s)$ with $i \in J$. Furthermore we only need the dissipation which occurs within the end grains 
$G_{i}(t), i \in J$. We thus define $\partial^{J} \mathcal{G}_{s}=\bigcup_{i \in J}\left(G_{i}(t) \cap \partial G_{i}(s)\right)$.

$$
\begin{aligned}
& L(t) \geq \frac{1}{\Lambda} \int_{0}^{t} \sqrt{\int_{\partial \mathcal{G}_{s}} v(x, s)^{2} d \mathcal{H}^{1}\left\llcorner\partial \mathcal{G}_{s}(x)\right.} d s \\
& \geq \frac{1}{\Lambda} \int_{0}^{t} \sqrt{\int_{\partial^{J} \mathcal{G}_{s}} v(x, s)^{2} d \mathcal{H}^{1}\left\llcorner\partial^{J} \mathcal{G}_{s}(x)\right.} d s,
\end{aligned}
$$

using the Cauchy-Schwarz inequality we obtain

$$
\geq \frac{1}{\Lambda} \int_{0}^{t} \frac{1}{\sqrt{\sum_{i \in J} \operatorname{Per}\left(G_{i}(t) \cap G_{i}(s)\right)}} \sum_{i \in J} \int_{G_{i}(t) \cap \partial G_{i}(s)}|v(x, s)| d \mathcal{H}^{1}\left\llcorner_{G_{i}(t) \cap \partial G_{i}(s)}(x) d s,\right.
$$

using (25)

$$
\geq \frac{1}{\Lambda} \int_{0}^{t} \frac{1}{\sqrt{\sum_{i \in J} \operatorname{Per}\left(G_{i}(t)\right)}} \sum_{i \in J} \int_{G_{i}(t) \cap \partial G_{i}(s)}|v(x, s)| d \mathcal{H}^{1}\left\llcorner G_{i}(t) \cap \partial G_{i}(s)(x) d s,\right.
$$

using (22)

$$
\geq \frac{1}{\Lambda^{2}} \frac{1}{\sqrt{2 \overline{\mathcal{E}}(t)}} \sum_{i \in J}\left(\left|G_{i}(t)\right|-\left|G_{i}(0)\right|\right) \geq \frac{1}{\Lambda^{2}} \frac{1}{\sqrt{2 \overline{\mathcal{E}}(t)}} \sum_{i \in J} \frac{1}{2}\left|G_{i}(t)\right|,
$$

using (24)

$$
\geq \frac{1}{\sqrt{2}} \frac{1}{\sqrt{\overline{\mathcal{E}}(t)}} \frac{1}{4}
$$

which proves the desired estimate.

Theorem 4. Let $\mathcal{G}_{t}, t \geq 0$ be the evolution of grains by the gradient flow of Voronoi diagrams on the flat torus $[0, \Lambda]^{2}$. Assume that at $t=0$ for all $i=1, \ldots, N,\left|G_{i}\right| \leq 1$. Then for all $\sigma \in(1,2)$ there exists $c_{1}>0, c_{2}>0$, depending only on $\sigma$, such that for all $t \geq c_{2}$

$$
\int_{0}^{T} \overline{\mathcal{E}}\left(\mathcal{G}_{t}\right)^{\sigma} d t \geq c_{1} \int_{0}^{T}\left(t^{-\frac{1}{2}}\right)^{\sigma} d t
$$

We note that this is a time averaged version of the claim that $\ell \lesssim t^{1 / 2}$.

Remark. We note that the proof of the theorem does not actually depend on how the quadruple and higher-order vertices are resolved. In particular the result would still apply if instead of the gradient vector any admissible vector (as defined in Section 5.2 ) is chosen. Moreover if we assume that there are only finitely many times at which there are quadruple or higher-order vertices then the upper bound on coarsening is not affected by how the vertices are resolved.

Proof. The theorem relies on the framework of Kohn and Otto [20]. In particular the interpolation inequality needed is proved in Theorem 3 . The desired dissipation inequality is $-\dot{\mathcal{E}} \geq(\dot{L})^{2}$. If it can be shown, then the ODE argument of Kohn and Otto (and more precisely the one used in the proof of Proposition 1 in [27]) implies the desired result. Due to the length of the ODE argument and since we have nothing to add, we choose not to present it here, but refer the reader to [27] (or [20]). Regarding the dissipation inequality, we note that, due to the way $L$ is defined, an energy dissipation equality holds at all but finitely many times at which a grain disappears. More precisely, from 
the definition of the gradient flow of Voronoi diagrams and Lemma 1 it follows that if $t_{1}<t_{2}$ are such that there are no grain disappearances in the time interval $\left[t_{1}, t_{2}\right]$ then

$$
\mathcal{E}\left(t_{1}\right)-\mathcal{E}\left(t_{2}\right)=\int_{t_{1}}^{t_{2}} \int_{\partial \mathcal{G}_{t}} v(x, s)^{2} d \mathcal{H}^{1}\left\llcorner\partial \mathcal{G}_{t}(x) d t=\int_{t_{1}}^{t_{2}}(\dot{L}(t))^{2} d t .\right.
$$

At the times when a grain disappears it may happen that $\mathcal{E}$ is discontinuous. Namely while the area of the disappearing gain goes to zero its perimeter may stay bounded from below. When this grain disappears the total perimeter has a downward jump. The function $L(t)$ remains continuous. Fortunately the possibility of energy being discontinuous has been addressed in [28](Lemma 5). Namely, it was shown that elements needed in the proof of Proposition 1 of [27] still hold if $\mathcal{E}$ is allowed to have downward jumps. Consequently the conclusion of Proposition 1 holds and thus establishes the desired coarsening bound.

\section{NumERICS}

To give additional insight into the coarsening dynamics, we performed a number of numerical experiments. We evolved a Voronoi diagram from time 0 to time $t$. We start by describing the procedure when the configuration is well described by the centers of the Voronoi cells: $\left(x_{1}(s), \ldots, x_{n(s)}(s)\right)$. Each center $x_{h}(s) \in \mathbb{T}_{2}$ lies on the two-dimensional torus. We take $\mathbb{T}_{2}=[0,1]^{2}$, with periodic boundary conditions.

Time is discretized by a temporally varying time step $\delta t_{p}$, with $t_{p}=\sum_{q=1}^{p} \delta t_{q}$. This approach is chosen because the numerical stability of the update depends strongly on the configuration. When all centers are well separated, it is possible to take quite large time steps. In contrast, when multiple centers are very close to each other, as is observed to occur frequently in the simulation, small perturbations in the center positions may give rise to very large changes in the positions of the Voronoi vertices, and thus in the total interface length. The configuration $X\left(t_{p}\right)$ is advanced to the configuration $X\left(t_{p+1}\right)$ by a forward Euler iteration for (7):

$$
X\left(t_{p}\right)=X\left(t_{p-1}\right)-\delta t_{p} \operatorname{grad} \mathcal{E}\left(X\left(t_{p-1}\right)\right) .
$$

where $\operatorname{grad} \mathcal{E}\left(X\left(t_{p-1}\right)\right)$ is computed by $(10)$, with $D_{v} \mathcal{E}$ computed with respect to the Voronoi vertices $V\left(t_{p-1}\right)=F\left(X\left(t_{p-1}\right)\right)$.

After each time step, it is checked whether (1) the time step should be adjusted, (2) any centers should be removed, (3) the representation of centers (in standard or collapsed coordinates) needs to be changed, and (4) the connectivity of the Voronoi diagram has changed. These checks are handled as follows:

(1) Change of time step. Once the updated centers $X\left(t_{p}\right)$ have been computed, it is directly checked that $\mathcal{E}\left(X\left(t_{p}\right)\right)<\mathcal{E}\left(X\left(t_{p-1}\right)\right)$. If energy does decrease, it is further checked whether performing two iterations with half the time step gives a similar decrease in energy. Specifically, it is required that

$$
\frac{\left|\Delta \mathcal{E}_{1}-\Delta \mathcal{E}_{2}\right|}{\left|\Delta \mathcal{E}_{1}\right|}<C
$$

where $\Delta \mathcal{E}_{1}=\mathcal{E}\left(X\left(t_{p}\right)\right)-\mathcal{E}\left(X\left(t_{p-1}\right)\right)$ and $\Delta \mathcal{E}_{2}$ is the change in energy from $\mathcal{E}\left(X\left(t_{p-1}\right)\right)$ following two steps of size $\left(\frac{1}{2} \delta t_{p}\right)$. If either of these checks fail, $\delta t_{p}$ is halved, and the iteration is repeated. If ten consecutive steps are performed successfully, then the subsequent time step is set as $\delta t_{p+1}=2 \delta t_{p}$. Any time a center is removed, $\delta t$ is reset to its initial value, $\delta t_{0}$. To prevent the time step from becoming infintesimally small and 
causing the evolution to get stuck, we also set a lower bound $\delta t_{\min }$ on the time step and require $\delta t \geq \delta t_{\min }$ at all times.

(2) Removal of centers. The area $A_{h}\left(t_{p}\right)$ associated with each center $x_{h}\left(t_{p}\right)$ is computed. If $A_{h}\left(t_{p}\right)<A_{\min }$, the center $x_{h}\left(t_{p}\right)$ is removed. Observe that removing a center $x_{h}\left(t_{p}\right)$ (as long as $A_{h}\left(t_{p}\right)$ is small) is expected to decrease the energy $\mathcal{E}\left(X\left(t_{p}\right)\right.$ ). In the simulations performed, removal of small cells is never observed to increase the energy $\mathcal{E}(X)$.

(3) Change in coordinates. It must be determined which centers, if any, need to be represented by collapsed coordinates. Let

$$
\Sigma=\bigcup_{h=1, \ldots, N\left(t_{p}\right)} B_{\rho}\left(x_{h}\left(t_{p}\right)\right)
$$

the union of balls of radius $\rho$ centered at the centers $x_{h}\left(t_{p}\right)$. Partition the centers by membership in the connected components of $\Sigma$. Each part of the partition containing $n>1$ centers has its centers represented in the collapsed coordinates for $n$ points. This selection criteria allows for centers to change representation from standard to collapsed coordinates and back through time, and also to change class of collapsed center (e.g. from a collapsed 2-center to a collapsed 3-center or vice-versa).

(4) Voronoi diagram update. As the centers move from $X\left(t_{p-1}\right)$ to $X\left(t_{p}\right)$, the Voronoi diagram also evolves. For example, vertices $v_{i j k}\left(t_{p-1}\right)$ and $v_{i j \ell}\left(t_{p-1}\right)$ may be replaced by $v_{i k \ell}\left(t_{p}\right)$ and $v_{j k \ell}\left(t_{p}\right)$ in a classical "edge-flipping" topological change. Thus the Voronoi diagram is recomputed at each iteration and the updated vertex set $V\left(t_{p}\right)$ is used in the subsequent step.

Six simulations are performed. Each is initialized with 500 centers placed uniformly at random on $\mathbb{T}_{2}$. In these simulations, we set $\delta t_{0}=10^{-5}$ and set $\delta t_{\min }=10^{-7}$. We choose $C=1 / 10, A_{\min }=10^{-4}$, and $\rho=10^{-4}$. We require that $d \geq 10^{-8}$ in all collapsed coordinates to prevent the numerical system from becoming overly stiff. The simulations are run until only 40 centers remain.

Snapshots of the evolution from one of the simulations are shown in Figure 4. It can be observed that centers frequently cluster together, requiring the use of the collapsed coodinate systems locally. Collapse of four centers is a relatively rare event, and is not seen in any of these snapshots. A collapse of more than four centers is never required based on the change in coordinates criterion specified previously.

Refer to Figure 5 for the time evolution of the average grain area on linear and logarithmic axes. The thick red line is the ensemble average of the five simulations, while the thick black line is the linear function $\langle A\rangle=.1585 t$. The good fit of this guide line to the data suggests that the average grain area grows linearly in time under this evolution after a short transient period. These numerical results are in good agreement with the coarsening rate upper bound of Section 6 .

Acknowledgments. ME was supported in part by a Rackham Predoctoral Fellowship. DS is grateful to NSF (grant DMS-0908415) and FCT (grant UTA_CMU/MAT/0007/2009). The research was also supported by NSF PIRE grant OISE-0967140. The authors are thankful to the Center for Nonlinear Analysis (NSF grant DMS-0635983) for its support. 


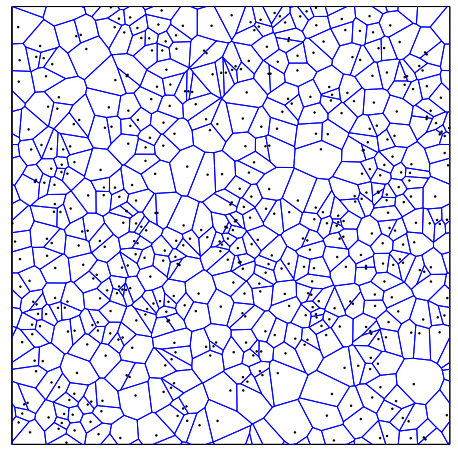

(a)

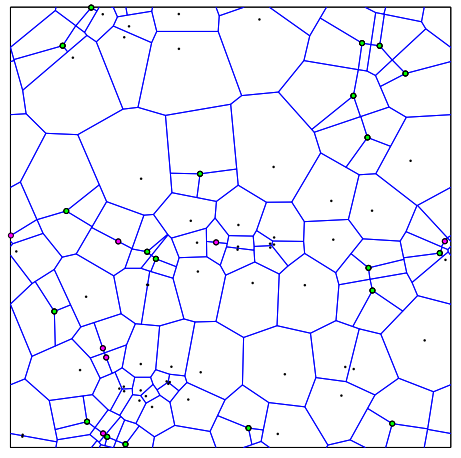

(d)

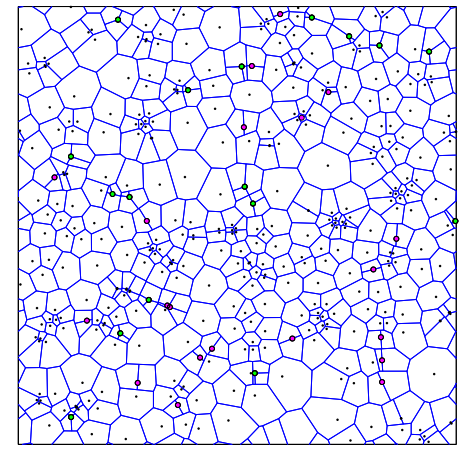

(b)

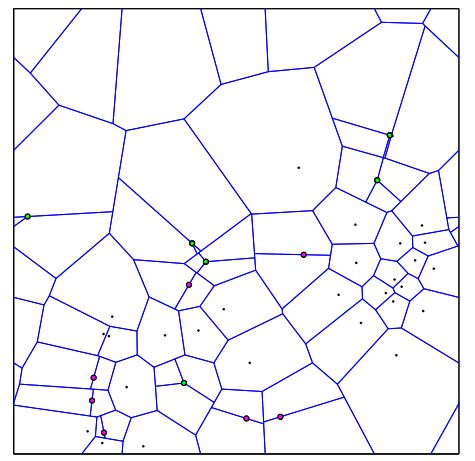

(e)

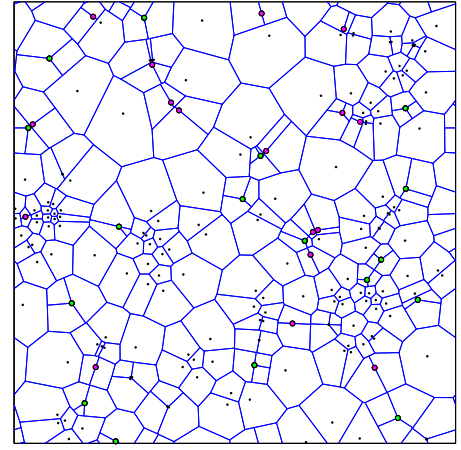

(c)

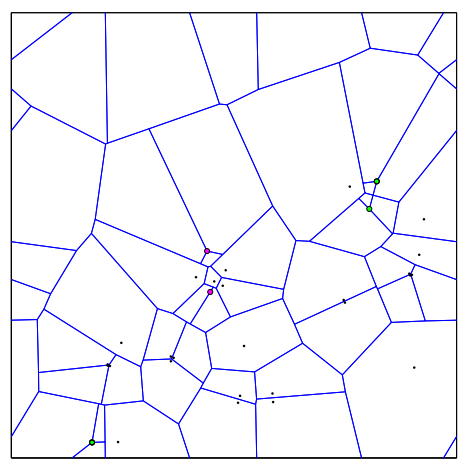

(f)

Figure 4. The microstructure from one simulation is shown (a) initially, and at (b) $t=2.87 \times 10^{-3}$, (c) $t=1.86 \times 10^{-2}$, (d) $t=4.04 \times 10^{-2}$, (e) $t=9.13 \times 10^{-2}$, (f) $t=1.21 \times 10^{-1}$, with $500,449,256,127,60$, and 40 grains remaining, respectively. The Voronoi diagram is drawn in blue, with isolated centers given by black dots. The location of two and three collided centers are denoted by magenta and green circles, respectively.

\section{Appendix A. Derivation of COORDinates For $v_{i j k}$}

Equation (13) may be derived as follows: The vertex $v_{i j k}$ must satisfy

$$
\left(x_{i}-v_{i j k}\right) \cdot\left(x_{i}-v_{i j k}\right)=\left(x_{j}-v_{i j k}\right) \cdot\left(x_{j}-v_{i j k}\right)=\left(x_{k}-v_{i j k}\right) \cdot\left(x_{k}-v_{i j k}\right),
$$

from which we subtract $v_{i j k} \cdot v_{i j k}$ to obtain

$$
x_{i} \cdot x_{i}-2 x_{i} \cdot v_{i j k}=x_{j} \cdot x_{j}-2 x_{j} \cdot v_{i j k}=x_{k} \cdot x_{k}-2 x_{k} \cdot v_{i j k},
$$

which can be combined to obtain

$$
2\left(x_{j}-x_{i}\right) \cdot v_{i j k}=x_{j} \cdot x_{j}-x_{i} \cdot x_{i}
$$

and

$$
2\left(x_{k}-x_{j}\right) \cdot v_{i j k}=x_{k} \cdot x_{k}-x_{j} \cdot x_{j} .
$$

In matrix form, this can be expressed as

$$
\left(\begin{array}{c}
\left(x_{j}-x_{i}\right)^{T} \\
\left(x_{k}-x_{j}\right)^{T}
\end{array}\right) v_{i j k}=\frac{1}{2}\left(\begin{array}{c}
x_{j} \cdot x_{j}-x_{i} \cdot x_{i} \\
x_{k} \cdot x_{k}-x_{j} \cdot x_{j}
\end{array}\right) .
$$




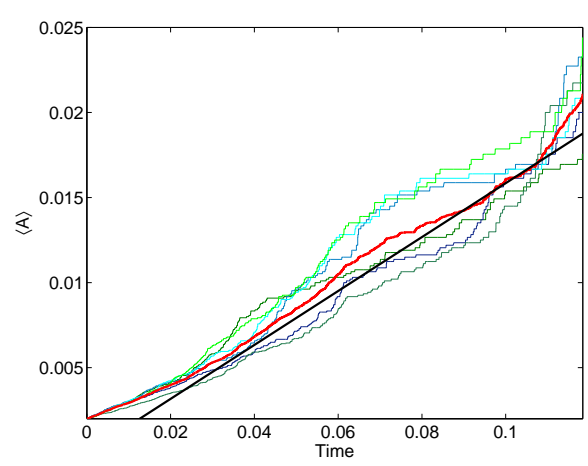

(a)

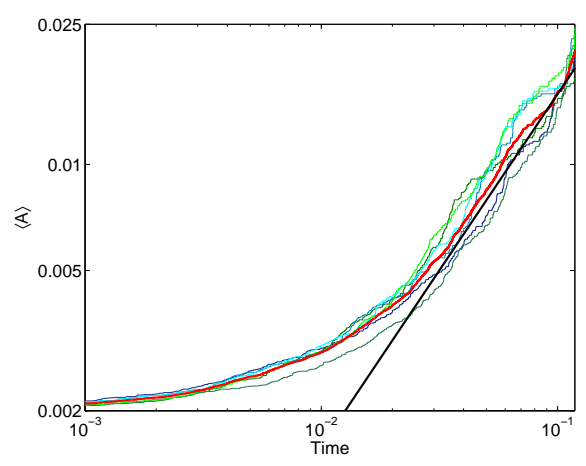

(b)

Figure 5. (a) Linear and (b) log-log plots of mean grain area $\langle A\rangle$ as a function of time, $t$. Six simulation runs are shown in shades of blue and green (thin lines), with ensemble average given by thick red line. For comparison, $\langle A\rangle=.1585 t$ is shown as thick black line, suggesting that $\langle A\rangle \propto t$, as expected.

We compute

$$
\left(\begin{array}{c}
\left(x_{j}-x_{i}\right)^{T} \\
\left(x_{k}-x_{j}\right)^{T}
\end{array}\right)^{-1}=\frac{\left(\left(x_{j}-x_{k}\right)^{\perp}\left(x_{j}-x_{i}\right)^{\perp}\right)}{\left(x_{j}-x_{i}\right) \cdot\left(x_{k}-x_{j}\right)^{\perp}} .
$$

(13) arises by left-multiplying (26) by (27), observing that $a \cdot b^{\perp}=-b \cdot a^{\perp}$, and collecting like terms.

\section{REFERENCES}

[1] M. P. Anderson, G. S. Grest, And D. J. Srolovitz, Computer simulation of normal grain growth in three dimensions, Philos. Mag. B, 59 (1989), pp. 293-329.

[2] M. P. Anderson, D. J. Srolovitz, G. S. Grest, And P. S. Sahni, Computer simulation of grain growth - I. Kinetics, Acta Metall., 32 (1984), pp. 783-791.

[3] K. Barmak, M. Emelianenko, D. Golovaty, D. Kinderlehrer, and S. TA'Asan, Towards a statistical theory of texture evolution in polycrystals, SIAM J. Sci. Comput., 30 (2008), pp. 31503169.

[4] P. BECK, Interface migration in recrystallization, in Metal Interfaces, R. Brick, ed., American Society for Metals, Cleveland, 1952, pp. 208-247.

[5] G. Bellettini, M. Chermisi, And M. Novaga, Crystalline curvature flow of planar networks, Interfaces Free Bound., 8 (2006), pp. 481-521.

[6] J. W. CAHN, Stability, microstructural evolution, grain growth, and coarsening in a two-dimensional two-phase microstructure, Acta Metall. Mater., 39 (1991), pp. 2189-2199.

[7] S. DAI, On the shortening rate of collections of plane convex curves by the area-preserving mean curvature flow, SIAM J. Math. Anal., 42 (2010), pp. 323-333.

[8] M. Elsey, S. Esedō̄lu, And P. SmerekA, Diffusion generated motion for grain growth in two and three dimensions, J. Comp. Phys., 228 (2009), pp. 8015-8033.

[9] - Large scale simulation of normal grain growth via diffusion generated motion, Proc. R. Soc. Lond. A, 467 (2011), pp. 381-401.

[10] V. E. FRADKov, A theoretical investigation of two-dimensional grain growth in the 'gas' approximation, Phil. Mag. Lett., 58 (1988), pp. 271-275.

[11] J. A. Glazier And D. Weaire, The kinetics of cellular patterns, J. Phys.: Condens. Matter, 4 (1992), pp. 1867-1894. 
[12] R. Henseler, B. Niethammer, and F. Otto, A reduced model for simulating grain growth, in Free boundary problems (Trento, 2002), vol. 147 of Internat. Ser. Numer. Math., Birkhäuser, Basel, 2004, pp. 177-187.

[13] C. Herring, Surface tension as a motivation for sintering, in The Physics of Powder Metallurgy, W. Kingston, ed., McGraw-Hill, New York, 1951, pp. 143-179.

[14] - The use of classical macroscopic concepts in surface-energy problems, in Structure and Properties of Solid Surfaces, R. Gomer and C. Smith, eds., University of Chicago Press, Chicago, 1953, pp. 5-72.

[15] M. Herrmann, P. Laurençot, and B. Niethammer, Self-similar solutions to a kinetic model for grain growth, J. Nonlinear Sci., 22 (2012), pp. 399-427.

[16] D. Joyce, On manifolds with corners, in Advances in Geometric Analysis, S. Janeczko, J. Li, and D. Phong, eds., International Press, Boston, 2012, pp. 225-258.

[17] D. Kinderlehrer And C. Liu, Evolution of grain boundaries, Math. Models Methods Appl. Sci., 11 (2001), pp. 713-729.

[18] D. Kinderlehrer, I. Livshits, And S. TA'Asan, A variational approach to modeling and simulation of grain growth, SIAM J. Sci. Comput., 28 (2006), pp. 1694-1715.

[19] D. A. Klain and G.-C. Rota, Introduction to geometric probability, Lezioni Lincee. [Lincee Lectures], Cambridge University Press, Cambridge, 1997.

[20] R. V. Kohn And F. Отто, Upper bounds on coarsening rates, Comm. Math. Phys., 229 (2002), pp. 375-395.

[21] R. Lindenbergh, W. van der Kallen, And D. Siersma, Configuration spaces and limits of Voronoi diagrams, in Geometry and Topology of Caustics - CAUSTICS '02, vol. 62 of Banach Center Publ., Polish Acad. Sci., Warsaw, 2004, pp. 183-195.

[22] R. C. Lindenbergh, Limits of Voronoi diagrams, Rijksuniversiteit te Utrecht, Utrecht, 2002. Dissertation, Universiteit Utrecht, Utrecht, 2002.

[23] R. D. MacPherson And D. J. Srolovitz, The von Neumann relation generalized to coarsening of three-dimensional microstructures, Nature, (2007), p. 10531055.

[24] P. W. Michor And D. Mumford, Vanishing geodesic distance on spaces on spaces of submanifolds and diffeomorphisms, Doc. Math, 10 (2005), pp. 217-245.

[25] L. Mugnai And C. Seis, On the Coarsening Rates for Attachment-Limited Kinetics, SIAM J. Math. Anal., 45 (2013), pp. 324-344.

[26] W. W. Mullins, Two-dimensional motion of idealized grain boundaries, J. Appl. Phys., 27 (1956), pp. 900-904.

[27] F. Отto, T. Rump, And D. SlepČEv, Coarsening rates for a droplet model: rigorous upper bounds, SIAM J. Math. Anal., 38 (2006), pp. 503-529 (electronic).

[28] D. SlepČEv, Coarsening in nonlocal interfacial systems, SIAM J. Math. Anal., 40 (2008), pp. 10291048.

[29] J. von Neumann, in Metal Interfaces, C. Herring, ed., American Society for Metals, Cleveland, 1952, pp. 108-110.

[30] F. Wakai, N. Enomoto, And H. OGawa, Three-dimensional microstructural evolution in ideal grain growth - general statistics, Acta Mater., 48 (2000), pp. 1297-1311.

Courant Institute of Mathematical Sciences, New York University; Department of Mathematical Sciences, Carnegie Mellon University 